\title{
Halitosis: A Review of Etiology and Management
}

\author{
${ }^{1}$ Dr. Praveenkumar Ramdurg M.D.S ${ }^{2}$ Dr. Vijaylaxmi Mendigeri ${ }_{\text {M.D.S }}$ \\ ${ }^{I}$ Assistant Professor Department of Oral Medicine and Radiology P.M.N.M Dental College and Hospital, \\ BagalkotKarnataka \\ ${ }^{2}$ Asst Proff Department of Orthodontics P.M.N.M Dental College and Hospital, Bagalkot Karnataka
}

\begin{abstract}
Bad breath or "Oral halitosis" is a frequent or persistent unpleasant odor of breath. It is a common and multi-factorial condition. In around 85 percent of cases, halitosis is the result of microbial activity in the mouth. In patients with healthy periodontal tissue, causative bacteria deposit on the dorsal tongue. The basic psychopathological process is manifested by the release of substances caused by degradation due to bacterial activity, such as volatile sulfur compounds (VSC), organic acids, and products of metabolic activity. Factors contributing to the overgrowth of causative bacteria include decreased salivary flow and stress. This presentation reviews the current knowledge of etiology and different aspects of its diagnosis and treatment.
\end{abstract}

Key words: Halitosis, Volatile sulfur compounds, Tongue coating, Mouth rinses, Chlorhexidine.

\section{Introduction}

Have you ever tried to smell your own breath? It's a common myth people use, but deprived of any truth. It is impossible to smell your own breath by cupping your hand up to your nose and smelling. Because our nose and mouth are not separate organs, and because our nose tends to filter out our own smells, smelling our own breath is practically impossible... Every day, while many develop faulty perceptions about having bad breath that affect their entire lives, others who have halitosis are unaware of their condition: this is called the "Bad breath Paradox".

Halitosis, fetor oris, oral malodor or bad breath are the general terms used to describe unpleasant breath emitted from a person's mouth regardless of whether the odorous substances in the breath originate from oral or non-oral sources ${ }^{1}$. Halitosis is an oral health condition characterized by consistently emanating odorous breath and may be caused by several agents including certain foods, poor oral health care, improper cleaning of dentures, decreased salivary flow rate, tobacco products or a medical condition. In $90 \%$ of cases, though, the causes of halitosis are located in the mouth and can be attributed to deep carious lesions, periodontal disease, oral infections, periimplant disease, pericoronitis, mucosal ulcerations, impacted food or debris, factors causing decreased salivary flow rate and, mainly, tongue coating ${ }^{2}$.

The tongue is a major site of oral malodor production, while periodontal disease and other factors seem to be only a fraction of the overall problem ${ }^{3}$. In addition; current social norms emphasize the importance of personal image and interpersonal relationships. Thus, halitosis may be an important factor in social communication and, therefore, may be the origin of concern not only for a possible health condition but also for frequent psychological alterations leading to social and personal isolation ${ }^{4}$. Although oral malodor or bad breath is an unpleasant condition experienced by most individuals, it typically results in transient discomfort.

\section{History}

"Fetor oris", "mauvaise haleine", the universal medical term "halitosis" used since 1930 comes from "Halitus" meaning "breath", and "-osis" meaning "chronic disorder". Now if the term "halitosis" is relatively recent, its negative effects go way back to the most ancient times of humanity. In Talmudic Law (2000 years old), mouth malodor of the partner may constitute a founded motive for divorce. Chinese emperors in Old China used to ask their visitors to chew clove before personal meetings ${ }^{5}$. Ebert's Papyrus (around 1700 BC) mentions a medication used in Old Egypt to alleviate bad breath: the tablets are made out of a cocktail based on incense, cinnamon, myrrh, and honey. Hippocrates (460-377 BC) had an exotic recipe based on marble powder for women suffering from bad breath. Old traditional treatments used Guava leafs in Thailand, eggshells in China, parsley in Italy, and urine-based mouth rinse in some European cultures ${ }^{6}$.

\section{Halitosis and the presence of oral microorganisms}

The oral microorganisms most likely to cause oral malodor are Gram-negative bacteria species including Treponema denticola, Porphyromonas gingivalis, Porphyromonas endodontalis, Prevotella intermedia, Bacteroides loescheii, Enterobacteriaceae, Tannerella forsythensis, Centipeda periodontii, Eikenella corrodens, Fusobacterium nucleatu ${ }^{7}$. 
The agents that give rise to oral malodor include especially the volatile sulfide compounds, diamines, and short chain fatty acids ${ }^{8}$. The principal components of bad breath are volatile sulfide compounds (VSC), especially hydrogen sulfide, methyl mercaptan, and dimethylsulfide ${ }^{10}$ or compounds such as butyric acid, propionic acid, putrescine, and cadaverine ${ }^{9}$. These compounds result from the proteolytic degradation by predominantly anaerobic Gram negative oral microorganisms of various sulfur-containing substrates in food debris, saliva, blood, and epithelial cells ${ }^{10}$.

\section{Classification And Etiology Of Halitosis}

When dealing with the problem of halitosis or with halitosis patient, it is important to distinguish between "genuine halitosis" and pseudo-halitosis". "Genuine halitosis" is where the breath malodor is a real problem that can be easily diagnoses either by organoleptic or by physic-chemical means. "Pseudo- halitosis" is where the oral malodor does not exist, but the patient believes that he or she has it. If after successful treatment for either genuine halitosis or pseudo-halitosis the patient still believes that he or she has halitosis, and then diagnosis termed "halitophobia"11

Genuine halitosis is sub classified as physiologic or pathologic halitosis (Table 1). Physiologic halitosis, also termed transient halitosis, has its origin in the dorsum of the tongue, is self limiting, and does not prevent the patient from carrying out a normal life. On the contrary, pathologic halitosis is permanent, does not resolve by usual oral hygiene methods, and prevent the patient from carrying out a normal life.

\section{Diagnosis}

It is important to understand whether a given complaint of bad breath is justified and whether the odor originates in the mouth, nose or elsewhere. A full history and an oral examination will be required. The first step in assessment is to determine whether halitosis is present. This is important as most individuals are poor judges of their own breath odor.

The clinical assessment of oral malodor is usually subjective and is based on smelling the exhaled air of the mouth and nose and comparing the two (organoleptic assessment). Odor detectable from the mouth but not from the nose is likely to be of oral or pharyngeal origin. Odor from the nose alone is likely to be coming from the nose or sinuses ${ }^{12}$. In rare instances when the odor from the nose and mouth are of similar intensity, a systemic cause of the malodor may be likely (Table 2).

Assessment of the quality of the odor (the hedonic method) relies on the use of trained clinical judges ${ }^{13,14}$. Objective measurement of the breath components is rarely used in routine clinical practice, as it is expensive and time consuming. Volatile sulphur compounds can be measured by using a portable sulphide monitor, but as oral malodor may comprise agents other than volatile sulphur compounds this may provide an inaccurate assessment of the source and intensity of oral malodor. Gas chromatography of oral breath is a potential method of determining the components of oral malodor, but this is not of practical clinical application $^{15}$.

\section{Management}

The management of halitosis starts by taking a detailed history of the condition, duration, severity, and impact on the patient's everyday life. Examination involves clinical, radiographic, and special tests. The contributing medical conditions, once identified, are referred for treatment accordingly. Clinical examination checks the patient's oral hygiene, caries, and periodontal status; plaque retention factors are also recorded. Radiographic examination should look for evidence of dental caries, alveolar bone defects, and defective restorations.

Successful treatment of halitosis depends on a correct diagnosis and the implementation of a causerelated therapy ${ }^{11}$. After a positive diagnosis for oral halitosis has been made, the treatment plan is implemented, which comprises elimination of the causative agent and improvement of the oral health status ${ }^{16}$. Although the multiple possible etiologies include oral and non-oral causes, the majority of breath malodor cases originate from the oral cavity. Briefly, the treatment of oral malodor can therefore be focused on the reduction of the intraoral bacterial load.

Miyazaki et al. ${ }^{17}$ established the recommended examination for halitosis and a classification of halitosis with corresponding treatment needs. Accordingly, different treatment needs (TN) have been described for the various diagnostic categories. The responsibility for the treatment of physiologic halitosis (TN-1), oral pathologic halitosis (TN-1 and TN-2), and pseudo-halitosis (TN-1 and TN-4) resides on dental practitioners. However, extra-oral pathologic halitosis $(\mathrm{TN}-3)$ and halitophobia $(\mathrm{TN}-5)$ should be managed by a physician or medical specialist and a psychiatrist or psychological specialist. Table- 3 describes the 5 different categories of treatment needs according to diagnosis. 


\section{Reduction in total load of oral microorganisms and or bacterial nutrients in the oral cavity Mechanical approach}

Several studies have implicated the dorsum of the tongue as the primary source of volatile sulphur compound (VSC), both in periodontally diseased and healthy individuals ${ }^{2,18,19}$. Mechanical reduction of malodor and of the intraoral bacterial count may be achieved by disrupting the tongue biofilm, thus decreasing the production of VSCs and other volatile organic compounds ${ }^{20}$. Researchers have been able to find positive correlations between tongue coating status (amount and or presence) and the different parameters directly related with oral malodor. In this scenario, the tongue becomes the most important microenvironment to study and to target in the prevention and treatment of oral halitosis and also as a potential reservoir for periodontal pathogens. Various mechanical approaches to reduce load of oral microorganism are:

1. Gently cleaning the tongue surface: Twice daily is the most effective ways to keep bad breath in control; that can be achieved using tooth brush, tongue cleaner or tongue brush/scraper to wipe off the bacterial biofilm, debris, and mucus ${ }^{21}$. An inverted teaspoon may also do the job. Scraping or otherwise damaging the tongue should be avoided and scraping of V-shaped row of taste buds found at the extreme back of tongue should slao be avoided.

2. Eating a healthy breakfast: Rough foods help to clean the very back of the tongue.

3. Chewing gum: since dry-mouth can increase bacterial build up and cause or worsen bad breath, chewing sugarless gum can help with the production of saliva, and thereby help to reduce bad breath. Chewing may help particularly when mouth is dry, or when cannot perform oral hygiene procedures after meals. These aids in provision of saliva, which washes away oral bacteria, and promotes mechanical activity which helps, cleanse the mouth ${ }^{22}$.

4. Gargling: Gargling right before bedtime with a plain water or salt water is effective. Several types of commercial mouthwashes have been shown to reduce malodor for hours.

5. Maintaining proper oral hygiene: this includes daily tongue cleaning, flossing and periodic visits to dentists and hygienists. Flossing is particularly important in removing rotting food debris and bacterial plaque from between the teeth, especially at the gum line ${ }^{23}$. Dentures should be properly cleaned and soaked overnight in antibacterial solution.

6. Traditional remedies: according to traditional Ayurvedic medicine, chewing areca nut and betel leaf is an excellent remedy against bad breath.

\section{Chemical approach}

The goal of any antimicrobial treatment would be to reduce the proteolytic, anaerobic flora found on the tongue surface. Mouth rinses with antimicrobial properties can reduce oral malodor by reducing the number of microorganisms chemically. Often used active ingredients in these products are chlorhexidine ( $\mathrm{CHX})$, essential oils (EOs), triclosan and cetylpyridinium chloride (CPC). Mouth rinses can also reduce halitosis by chemically neutralizing odor compounds, including VSCs.

\section{Chlorhexidine}

CHX gluconate is a cationic bis-biguanide, with a very broad antimicrobial spectrum. Being the most studied antimicrobial agent in the treatment of gingivitis, it has also been tested for its efficacy in the treatment of oral halitosis. Results from a case series study in halitosis patients suggested a significant effect of $\mathrm{CHX}$ rinsing and tongue brushing after 1 week of treatment ${ }^{24,25}$.In several studies, a $0.2 \% \mathrm{CHX}$ mouthrinse produced significant reductions in volatile sulfur-containing compound levels and in organoleptic scores ${ }^{26,27}$. Similar results with

$0.12 \% \mathrm{CHX}$-(di) gluconate were reported in combination with teeth and tongue brushing ${ }^{24,25}$.

Although being considered the gold standard mouthrinse for halitosis treatment, CHX has undesirable side effects. The safety of an effective agent that might be used repeatedly needs to be established. An agent is needed that approaches the clinical efficacy of CHX but with better safety and comfort features.

\section{Triclosan}

The clinical experiments performed by Young et al. ${ }^{28}$ showed that mouth-rinsing with triclosan solubilized in sodium lauryl sulfate, propylene glycol and water gave a marked and long-lasting anti-VSC effect. Triclosan and CPC mouth rinses were more effective in reducing bad breath than in reducing supra gingival plaque accumulation. Therefore, it could be postulated that the superior reducing effect of these specific mouth rinses on bad breath may be related primarily to their efficacy in reducing the load of VSC-related microorganisms and oral debris in the whole mouth niches rather than only in supra gingival plaque reduction ${ }^{27}$. 


\section{Essential oils}

Essential oils, including hydro-alcohol solutions of thymol, menthol, eucalyptol, and methyl salicylate, have been used in mouthwashes to prevent periodontal disease. Anti-plaque and anti-gingivitis activity has been demonstrated in several studies ${ }^{29}$. Rinsing with an EOs mouth rinse can have long lasting effects in reducing anaerobic bacteria overall as well as Gram-negative anaerobes and VSC producing bacteria. The significant reductions in numbers of these bacteria produced by the EO mouth rinse, both in plaque and on the dorsum of the tongue, can play a key role in explaining the EO mouth rinse's effectiveness in reducing supra gingival plaque and gingivitis as well as its effectiveness in controlling intrinsic oral malodor throughout the test period of 14 days $^{30}$.

Zinc

Metals such as zinc, sodium, tin and magnesium are thought to interact with sulfur. The mechanism proposed is that metal ions oxidize the thiol groups in the precursors of volatile sulfur-containing compounds ${ }^{31}$.Morning breath odor can be successfully reduced by the sole use of an amine fluoride-stannous fluoride-containing mouth rinse twice daily, which significantly reduces the bacterial load in the saliva and retards the de novo plaque formation ${ }^{32}$. Unfortunately, both cupric and stannous ions have the potential to discolor teeth, either as a result of sulfide formation on the teeth after extended periods of use or due to the precipitation of dietary chromogen.

\section{Effective combination of agents \\ Chlorhexidine and zinc}

A CHX and zinc mouth rinse had a strong effect on volatile sulfur-containing compounds and was effective for at least 9 hours. Control rinses with $\mathrm{CHX}$ or zinc alone had a moderate and strong effect for 1 hour, but this effect diminished with time, respectively, fast and slightly ${ }^{33}$.

\section{Cetylpyridinium and zinc ions}

A CPC and zinc mouth rinse had a good synergistic effect on volatile sulfur-containing compounds levels after 1 hour, but minimally above the effect of zinc alone ${ }^{33}$.

\section{Chlorhexidine, cetylpyridinium chloride and zinc-lactate}

Chlorhexidine is still the gold standard mouth rinse, but it does have some side effects. Due to these disadvantages, new formulations have been developed. Since CHX and CPC are both antimicrobial agents, it seems reasonable to assume that the new marketed mouthwash that contains CHX and CPC acts by reducing the number of VSC-producing bacteria on the dorsum of the tongue. Moreover, zinc-lactate, besides its antimicrobial activity, may reduce VSC scores by transforming them into insoluble compounds ${ }^{34}$.

\section{Conclusion}

The present review demonstrated that halitosis is a common problem impacting individuals at all ages. The main etiological factors include bacteria in the oral cavity related especially to periodontal disease and the dorsum of the tongue. Medical aspects include ear-nose-throat and gastroenterological problem. It is clear that a successful treatment of halitosis involves an appropriate diagnosis, professional therapy, mechanical plaque control, including tooth brushing and tongue cleaning, possibly combined with the use of an effective antimicrobial mouth rinse.

\section{Acknowledgement:}

\section{References:}

[1]. Innocent- Ituah I. Halitosis: hindrance or hint? J Miss State Med Assoc 2009 Dec; 50(12): 422-25

[2]. van den Broek AM, Feenstra L, de Baat C. A review of the current literature on management of halitosis. Oral Dis. 2008; 14(1):309.

[3]. Rosenberg M. Bad breath and periodontal disease: how related are they? J Clin Periodontol. 2006; 33(1):29-30.

[4]. Sanz M, Roldán S, Herrera D. Fundamentals of Breath Malodour. J Contemp Dent Pract. 2001 Nov 15; $2(4): 1-17$.

[5]. Talmud de Babylone. Traité Ketubot, p.72-77a, New York, Mesorah Publications, 1998

[6]. Anthologie Grecque., 11 241; 11 247; 11415, Paris, Editions Les Belles Lettres, 2003.

[7]. Awano S, Gohara K, Kurihara E, Ansai T, Takehara T. Therelationship between the bacteria in saliva and halitosis. Int Dent J. 2002;52 Suppl 3:212-6.

[8]. Porter SR, Scully C. Oral malodour (halitosis). BMJ. 2006;23;333(7569):632-5. Review.

[9]. Goldberg S, Kozlovsky A, Gordon D, Gelernter I, Sintov A, Rosenberg M. Cadaverine as a putative component of oral malodor. J Dent Res. 1994;73(6):1168-72.

[10]. Tonzetich J. Production and origin of oral malodor: a review of mechanisms and methods of analysis. J Periodontol. 1977;48(1):1320.

[11]. Yaegaki K, Coil JM. Examination, classification, and treatment of halitosis; clinical perspectives. J Can Dent Assoc. 2000 May;66(5):257-61. Review. 
[12]. Rosenberg M, McCullough CA. Measurements of oral malodor: current methods and future prospects. J Periodontol 1992;63:77682.

[13]. Greenman J, Duffield J, Spencer P, Rosenberg M, Corry D, Saad S, et al. Study on the organleptic intensity scale for measuring oral malodor. J Dent Res 2004;83:81-5.

[14]. Nachnani S, Majerus G, Lenton P, Hodges J, Magallanes E. Effects of training on odor judges scoring intensity. Oral Dis 2005;11:40-4.

[15]. Yaegaki K, Coil JM. Clinical dilemmas posed by patients with psychosomatic halitosis. Halitophobia 1999;30:328-33.

[16]. Lee PP, Mak WY, Newsome P. The aetiology and treatment of oral halitosis: an update. Hong Kong Med J. 2004;10(6):414-8

[17]. Miyazaki H, Arao M, Okamura K, Kawaguchi Y, Toyofuku A, Hoshi K et al. [Tentative classification of halitosis and its treatment needs] [Article in Japanese]. Niigata Dent J. 1999;32:7-11.

[18]. Yaegaki K, Sanada K. Volatile sulfur compounds in mouth air from clinically healthy subjects and patients with periodontal disease. J Periodontal Res. 1992;27:233-8.

[19]. Roldán S, Winkel EG, Herrera D, Sanz M, Van Winkelhoff AJ. The effects of a new mouthrinse containing chlorhexidine, cetylpyridinium chloride and zinc lactate on the microflora of oral halitosis patients: a dual-centre, double-blind placebocontrolled study. J Clin Periodontol. 2003;30(5):427-34.

[20]. Krespi YP, Shrime MG, Kacker A. The relationship between oral malodor and volatile sulfur compound-producing bacteria. Otolaryngol Head Neck Surg. 2006;135(5):671-6.

[21]. Yaegaki K, Coil JM, Kamemizu T, Miyazaki H. Tongue brushing and mouth rinsing as basic treatment measures for halitosis. Int Dent J. 2002;52 Suppl 3:192-6.

[22]. Rosing CK, Gomes SC, Bassani DG, Oppermann RV. Effect of chewing gums on the production of volatile sulfur compounds (VSC) in vivo. Acta Odontol Latinoam 2009 June(1):11-4

[23]. Tanaka M, Anguri H, Nishida N, Ojima M, Nagata H, Shizukuishi S. Reliability of clinical parameters for predicting the outcome of oral malodor treatment. J Dent Res. 2003;82:518- 22.

[24]. Bosy A, Kulkarni GV, Rosenberg M, McCulloch CA. Relationship of oral malodor to periodontitis: evidence of independence in discrete subpopulations. J Periodontol. 1994;65(1):37-46.

[25]. De Boever EH, Loesche WJ. Assessing the contribution of anaerobic microflora of the tongue to oral malodor. J Am Dent Assoc. 1995;126(10):1384-93

[26]. van Steenberghe D, Avontroodt P, Peeters W, Pauwels M, Coucke W, Lijnen A et al. Effect of different mouthrinses on morning breath. J Periodontol. 2001;72(9):1183-91

[27]. Carvalho MD, Tabchoury CM, Cury JA, Toledo S, Nogueira- Filho GR. Impact of mouthrinses on morning bad breath in healthy subjects. J Clin Peridontol. 2004;31:85-90.

[28]. Young A, Jonski G, Rölla G. A study of triclosan and its solubilizers as inhibitors of oral malodour. J Clin Periodontol. 2002;29:1078-81.

[29]. Gunsolley JC. A meta-analysis of six-month studies of antiplaque and antigingivitis agents. J Am Dent Assoc. 2006 Dec;137(12):1649-57. Review.

[30]. Fine DH, Furgang D, Sinatra K, Charles C, McGuire A, Kumar LD. In vivo antimicrobial effectiveness of an essential oilcontaining mouth rinse $12 \mathrm{~h}$ after a single use and 14 days' use. J Clin Periodontol. 2005;32:335-40.

[31]. $\mathrm{Ng} \mathrm{W}$, Tonzetich J. Effect of hydrogen sulphide and methyl mercaptan on the permeability of oral mucosa $\mathrm{J}$ Dent Res. 1984;63:994-7.

[32]. Quirynen M, Avontroodt P, Soers C, Zhao H, Pauwels M, Coucke W et al. The efficacy of amine fluoride/stannous fluoride in the suppression of morning breath odour. J Clin Periodontol. 2002;29:944-54.

[33]. Young A, Jonski G, Rölla G. Inhibition of orally produced volatile sulfur compounds by zinc, chlorhexidine or cetylpyridinium chloride - effect of concentration. Eur J Oral Sci. 2003;111:400-4.

[34]. Winkel EG, Roldán S, Van Winkelhoff AJ, Herrera D, Sanz M. Clinical effects of a new mouthrinse containing chlorhexidine, cetylpyridinium chloride and zinc-lactate on oral halitosis. A dual-center, double-blind placebo-controlled study. J Clin Periodontol. 2003;30:300-6.

Table I-Causes of halitosis

\begin{tabular}{|c|c|}
\hline Physiologic & Pathologic \\
\hline $\begin{array}{l}\text { Lack of flow of saliva during sleep } \\
\text { Food } \\
\text { Smoking } \\
\text { Menstruation }\end{array}$ & $\begin{array}{l}\text { Disorders of the oral cavity } \\
\text { Poor oral hygiene } \\
\text { Dental plaque } \\
\text { Dental caries } \\
\text { Gingivitis } \\
\text { Stomatitis } \\
\text { Periodontitis } \\
\text { Hairy tongue } \\
\text { Oral carcinoma } \\
\text { Disorders of upper respiratory tract } \\
\text { Breathing through the mouth } \\
\text { Chronic sinusitis } \\
\text { Foreign bodies } \\
\text { Atrophic rhinitis } \\
\text { Wegener's granulomatosis } \\
\text { Gastrointestinal conditions } \\
\text { Salivary gland dysfunction } \\
\text { Dehydration } \\
\text { Anticholinergic drugs } \\
\text { Radiotherapy }\end{array}$ \\
\hline
\end{tabular}




\begin{tabular}{|l|l|}
\hline & Sjogren's syndrome \\
& Peritonsillar abscess \\
Retropharyngeal abscess & Vincent's angina \\
Carcinoma of the tonsil or pharynx & \\
& Systemic diseases \\
Leukemia & Agranulocytosis \\
& Febrile illness with dehydration \\
Ketoacidosis \\
Hepatic failure \\
Azotemia \\
& Drugs \\
& Lithium salts \\
& Penicillamine \\
& Griseofulvin \\
& Thiocarbamide \\
& Dimethylsulfoxide \\
\hline
\end{tabular}

Table 2: Clinical assessment of oral malodor

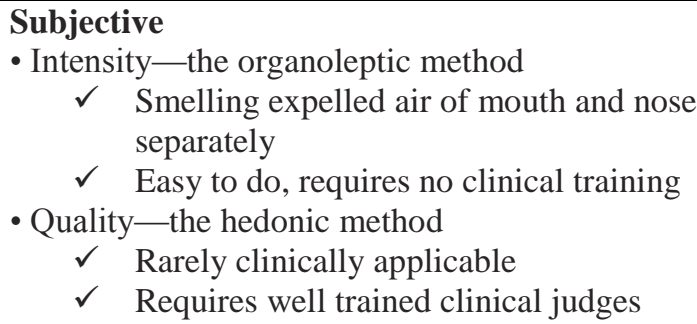

- Intensity - the organoleptic method

$\checkmark \quad$ Smelling expelled air of mouth and nose separately

Easy to do, requires no clinical training

- Quality - the hedonic method

$\checkmark$ Rarely clinically applicable

$\checkmark$ Requires well trained clinical judges

\section{Objective}

- Detection of sulphides with appropriate monitor-simple, but may fail to detect oral malodour caused by non-sulphide components

- Gas chromatography — not applicable for routine clinical practice

- Bacterial detection (such as benzoyl-arginine-naphthylamide test, polymerase chain reaction, dark field microscopy) — not applicable for routine clinical practice

Table 3 - Treatment needs (TN) for breath malodor divided in 5 categories.

\begin{tabular}{|c|l|}
\hline Category & \multicolumn{1}{c|}{ Description } \\
\hline TN-1 & $\begin{array}{l}\text { Explanation of halitosis and instructions for oral hygiene (support and reinforcement } \\
\text { of a patient's own self-care for further improvement of his/her oral hygiene). }\end{array}$ \\
\hline TN-2 & $\begin{array}{l}\text { Oral prophylaxis, professional cleaning and treatment of oral diseases, especially } \\
\text { periodontal diseases. }\end{array}$ \\
\hline TN-3 & Referral to a physician or medical specialist. \\
\hline TN-4 & $\begin{array}{l}\text { Explanation of examination data, further professional instruction, education and } \\
\text { reassurance. }\end{array}$ \\
\hline TN-5 & Referral to a clinical psychologist, psychiatrist or other psychological specialist. \\
\hline
\end{tabular}

